\title{
Inhibition of the long non-coding RNA MALAT1 suppresses tumorigenicity and induces apoptosis in the human ovarian cancer SKOV3 cell line
}

\author{
SHIPING LIU, XUAN JIANG, WEIHUA LI, DONGYAN CAO, KENG SHEN and JIAXIN YANG \\ Department of Obstetrics and Gynecology, Peking Union Medical College Hospital, Peking Union Medical College, \\ Chinese Academy of Medical Sciences, Beijing 100730, P.R China
}

Received March 4, 2015; Accepted March 18, 2016

DOI: $10.3892 / 01.2016 .4435$

\begin{abstract}
Metastasis-associated lung adenocarcinoma transcript 1 (MALAT1) is a 8,000 nucleotide-long, spliced non-coding RNA, which has been reported to be deregulated in several tumors. However, to the best of our knowledge, the role of MALAT1 in ovarian cancer has not been previously investigated. The aim of the present study was to investigate the effect of MALAT1 inhibition on the tumorigenity of SKOV3 cells. First, stable MALAT1-knockdown ovarian cancer cells and control cells were established using lentivirus-mediated artificial micro RNA interference in order to investigate the effect of MALAT1 inhibition on cell viability, clonability, migration, invasion and apoptosis in vitro. In addition, the effect of MALAT1 on cell growth in nude mice was assessed. To identify the possible targets of MALAT1, total RNA was extracted from MALAT1-knockdown cells and control cells and a microarray analysis was performed. The results showed that MALAT1 inhibition significantly suppressed tumorigenity in vitro and in vivo $(\mathrm{P}<0.01)$. Compared with the control cells, 921 genes in the MALAT1-knockdown cells were deregulated by at least two-fold. The results of the reverse transcription-quantitative polymerase chain reaction showed that 19 of the 20 genes selected for validation confirmed the deregulation indicated by the microarray analysis. The findings define a major oncogenic role for MALAT1, which may offer an attractive novel target for therapeutic intervention in ovarian cancer.
\end{abstract}

Correspondence to: Ms. Jiaxin Yang, Department of Obstetrics and Gynecology, Peking Union Medical College Hospital, Peking Union Medical College, Chinese Academy of Medical Sciences, 1 Shuaifuyuan Wangfujing, Dongcheng, Beijing 100730, P.R. China E-mail: yangjiaxin0078@163.com

Key words: ovarian cancer, long non-coding RNAs, metastasis-associated lung adenocarcinoma transcript 1

\section{Introduction}

Ovarian cancer is the most lethal gynecological malignancy, and $<40 \%$ of patients with ovarian cancer are cured. In 2015, it is estimated that 21,290 new cases and 14,180 mortalities will occur in association with this disease in the United States (1). Epidemiological studies have identified several risk factors of ovarian cancer, including nulliparity, hormone therapy and pelvic inflammatory disease (2-4). Initial treatment for ovarian cancer consists of surgical staging and cytoreduction, followed by systemic chemotherapy in the majority of patients. Given the high mortality rate of ovarian cancer, the identification of novel biomarkers and therapeutic targets has been a major research focus for a number of years.

Long non-coding RNA (lncRNA) is an RNA molecule that is $>200$ nucleotides long and is not translated into a protein. Although the current understanding of the role of lncRNAs is limited, increasing numbers of studies have indicated that lncRNAs may regulate gene expression at various levels, including chromatin modification, transcription and posttranscriptional processing (5-7). lncRNAs have been reported to control various cellular processes, including proliferation, apoptosis and invasion, and are implicated in human diseases, including various types of tumors.

Metastasis-associated lung adenocarcinoma transcript 1 (MALAT1), also known as non-coding nuclear-enriched abundant transcript 2, is a 8,000 nucleotide-long, spliced non-coding RNA, which is highly conserved in mammals. MALAT1 has been reported to be deregulated in several tumors, including non-small cell lung cancer, hepatocellular carcinoma, cervical cancer, bladder cancer and colorectal cancer (8-12). However, the role of MALAT1 in ovarian cancer has not been previously investigated.

The present study indicated that MALAT1 inhibition significantly suppressed tumorigenicity and induced apoptosis in SKOV3 cells. In addition, the knockdown of MALAT1 was indicated to alter the expression of a number of genes associated with cell proliferation, metastasis and apoptosis. To the best of our knowledge, the present study is the first to report an oncogenic role for MALAT1 in ovarian cancer. 
Table I. Sequences of the four microRNA oligonucleotides.

Oligonucleotide

Sequence

\begin{tabular}{|c|c|}
\hline \multicolumn{2}{|l|}{ KD1 } \\
\hline Forward & $\begin{array}{l}\text { 5'-TGCTGTCCACTTGATCCCAACTCATCGTTTTGGCCACTGACTGACGATGAGTTG } \\
\text { ATCAAGTGGA-3' }\end{array}$ \\
\hline Reverse & $\begin{array}{l}\text { 5-CCTGTCCACTTGATCAACTCATCGTCAGTCAGTGGCCAAAACGATGAGTTGGGA } \\
\text { TCAAGTGGAC-3' }\end{array}$ \\
\hline \multicolumn{2}{|l|}{ KD2 } \\
\hline Forward & $\begin{array}{l}\text { 5'-TGCTGCTTATCTGTTAACAGCTGCCTGTTTTGGCCACTGACTGACAGGCAGCTT } \\
\text { AACAGATAAG-3' }\end{array}$ \\
\hline Reverse & $\begin{array}{l}\text { 5'-CCTGCTTATCTGTTAAGCTGCCTGTCAGTCAGTGGCCAAAACAGGCAGCTGTTA } \\
\text { ACAGATAAGC-3' }\end{array}$ \\
\hline \multicolumn{2}{|l|}{ KD3 } \\
\hline Forward & $\begin{array}{l}\text { 5'-TGCTGTGTACTATCCCATCACTGAAGGTTTTGGCCACTGACTGACCTTCAGTGG } \\
\text { GGATAGTACA-3' }\end{array}$ \\
\hline Reverse & $\begin{array}{l}\text { 5'-CCTGTGTACTATCCCCACTGAAGGTCAGTCAGTGGCCAAAACCTTCAGTGATGG } \\
\text { GATAGTACAC-3' }\end{array}$ \\
\hline \multicolumn{2}{|l|}{ KD4 } \\
\hline Forward & $\begin{array}{l}\text { 5'-TGCTGTTCCTTAGTTGGCATCAAGGCGTTTTGGCCACTGACTGACGCCTTGATC } \\
\text { AACTAAGGAA-3' }\end{array}$ \\
\hline Reverse & $\begin{array}{l}\text { 5'-CCTGTTCCTTAGTTGATCAAGGCGTCAGTCAGTGGCCAAAACGCCTTGATGCCA } \\
\text { ACTAAGGAAC-3' }\end{array}$ \\
\hline
\end{tabular}

\section{Materials and methods}

Cell lines and cell culture. The human ovarian cancer SKOV3 cell line and $293 \mathrm{~T}$ cells were obtained from the American Type Culture Collection (Manassas, VA, USA). The two cell lines were cultured in Dulbecco's modified Eagle's medium (HyClone; GE Healthcare Life Sciences, Logan, UT, USA), supplemented with $10 \%$ fetal bovine serum (HyClone; GE Healthcare Life Sciences), $100 \mathrm{U} / \mathrm{ml}$ penicillin and $100 \mu \mathrm{g} / \mathrm{ml}$ streptomycin (Invitrogen; Thermo Fisher Scientific, Inc., Waltham, MA, USA) in standard culture conditions of $5 \% \mathrm{CO}_{2}$ at $37^{\circ} \mathrm{C}$.

Establishment of stable MALAT1-knockdown (KD) and negative control (NC) cells. Four microRNA (miRNA) oligonucleotides targeting MALAT1 (KD1, KD2, KD3 and KD4; Table I) were synthesized and inserted into the pcDNA6.2-GW/EmGFP-miR vector (Invitrogen; Thermo Fisher Scientific, Inc.) and used for transient transfections. The pcDNA6.2-GW/EmGFP-miR-Neg vector (Invitrogen; Thermo Fisher Scientific, Inc.) was used as negative control. Cells were maintained in $5 \% \mathrm{CO}_{2}$ at $37^{\circ} \mathrm{C}$ and harvested at 2 days post-infection. Reverse transcription-quantitative polymerase chain reactive (RT-qPCR) was performed in order to determine the efficiency of MALAT1 knockdown and to screen for the miRNA with the greatest knockdown efficiency, which was then used for subsequent experiments. pLenti6.3-MCS/V5DEST lentiviral vector was purchased from Shanghai R\&S Biotechnology Co., Ltd. (Shanghai, China). The pLenti6.3-EmGFP-MALAT1-miR and plenti-EmGFP-miR-Neg vectors were constructed using the pcDNA6.2-GW/EmGFP-miR and pcDNA6.2-GW/EmGFP-miR-Neg vectors with the greatest knockdown efficiency, respectively. To produce recombinant lentiviruses, the $293 \mathrm{~T}$ cells were co-transfected with lentivirus expression plasmids and packaging plasmid mix (Invitrogen; Thermo Fisher Scientific, Inc.) using POLOdeliverer ${ }^{\mathrm{TM}} 3000$ Transfection Reagent (Ruisai Inc., Shanghai, China). The SKOV3 cells were then infected with these lentiviruses at a multiplicity of infection of 30 , and selected with blasticidin (Invitrogen; Thermo Fisher Scientific, Inc.). At 2 weeks post-infection, cells were harvested and qRT-PCR was performed to determine the expression levels of MALAT1 in $\mathrm{KD}$ and NC cells.

$R N A$ extraction and $R T-q P C R$. Total RNA was extracted from the cell samples using the Trizol reagent (Invitrogen; Thermo Fisher Scientific, Inc.), according to the manufacturer's instructions. The RNA integrity was analyzed using the Agilent 2100 Bioanalyzer (Agilent Technologies Inc., Santa Clara, CA, USA). Each RT reaction consisted of $0.5 \mu \mathrm{g}$ RNA, $2 \mu \mathrm{l}$ PrimerScript Buffer, $0.5 \mu \mathrm{l}$ oligo(dT), $0.5 \mu \mathrm{l}$ random primers and $0.5 \mu \mathrm{l}$ PrimerScript RT Enzyme mix I (Takara, Otsu, Japan), in a total volume of $10 \mu \mathrm{l}$. Reactions were performed in a GeneAmp ${ }^{\circledR}$ PCR System 9700 (Applied Biosystems; Thermo Fisher Scientific, Inc.) for 15 min at $37^{\circ} \mathrm{C}$, followed by heat inactivation of reverse transcriptase for $5 \mathrm{sec}$ at $85^{\circ} \mathrm{C}$. The $10-\mu \mathrm{l}$ RT reaction mixture was then diluted 10 times in nuclease-free water and held at $-20^{\circ} \mathrm{C}$. qPCR was performed using the LightCycler ${ }^{\circledR} 480$ II Real-Time PCR instrument (Roche Diagnostics, Basel, Switzerland) with a total volume including $1 \mu \mathrm{l}$ cDNA, $5 \mu 12 \mathrm{X}$ 
Table II. Primers used in reverse transcription-quantitative polymerase chain reaction.

\begin{tabular}{lll}
\hline Gene symbol & \multicolumn{1}{c}{ Forward primer } & \multicolumn{1}{c}{ Reverse primer } \\
\hline MALAT1 & ATCAGACCACCACAGGTTTACAG & GACCATCCCAAAATGCTTCA \\
WISP2 & GCTTCTCTCCGACTTCC & TCTGTGTGCCTTCTCTCA \\
MUC16 & ATCATCAGCTATGACATCGAC & TCTTCTCAGTGGATAGGCTTA \\
ZEB2 & TTGGTGTACCAAGAGGCAA & GTGGAGAAGAGACTCGGT \\
MMP2 & ACAGAAGGACTCAGGTTG & CTGGATTCTGGAGAACAGATTT \\
MMP11 & CTGAGCAACTGGGCTGTA & TGTACCTGTGATCTGTCTTTCT \\
VEGFA & GGGCAAATATGACCCAGT & CAGGGAAACAGTTGGCTAA \\
PGF & CCTTGGAGGAGAGAGACC & CAACCAGTGCAAATGACTTAG \\
H19 & TTTCATCCTTCTGTCTCTTTGT & TAACAACTAGAAGCTGATGCAC \\
ERBB4 & AATAAATGCTAAACCAAGCGT & GCGTCACTCAGTGTGCTA \\
SNAI2 & TGCAGACCCATTCTGATGTAA & GGGACAAAGAGAGTTCCTAAG \\
E2F8 & CCCAAAGGGTCACAATTAGT & CATCTCTCACCTGCCTCATA \\
CDK2 & CCCTTGTTTGTCCCTTCTAC & GTGAACCACTGGACAGGATA \\
CDC20 & TGGATCAAAGAGGGCAACT & AAGGGTGTCCTCGTGAAA \\
ADRA1B & AAGAGAACCACCAAGAACCTA & ATGGGAACTGATCTTTCATCAA \\
RASGRP1 & GTTCCAAACTAAAGCCAATAGC & AAACAGAGCAGGGAACTAC \\
FGF1 & TTTCAACTTTAGAACCGGGTC & TCAAGTCAAGGTCACAGTGAG \\
BAX & AGATGTGGTCTATAATGCGTT & TCTAAGCTGGGTCTGCTAAC \\
FN1 & AAGATTCCCGAGAGTAAATCAT & TTGACCTGAACTGGTCTTTGTA \\
MTBP & GAAAGCCACAAACAGAACG & AGCTTTCACCAAGTGCTAA \\
ECT2 & ACAACTCATTTGATATGAAGCG & CTCCACGACGTACTCAGCG \\
GAPDH & TGTTGCCATCAATGACCCCTT & \\
\hline
\end{tabular}

MALAT1, metastasis-associated lung adenocarcinoma transcript 1; WISP2, WNT1 inducible signaling pathway protein 2; MUC16, mucin 16, cell surface associated; ZEB2, zinc finger E-box binding homeobox 2; MMP2, matrix metallopeptidase 2; MMP11, matrix metallopeptidase 11;VEGFA, vascular endothelial growth factor A; PGF, placental growth factor; H19, H19, imprinted maternally expressed transcript (non-protein coding); ERBB4, erb-b2 receptor tyrosine kinase 4; SNAI2, snail family zinc finger 2; E2F8, E2F transcription factor 8; CDK2, cyclin-dependent kinase 2; CDC20, cell division cycle 20; ADRA1B, adrenoceptor a 1B; RASGRP1, RAS guanyl releasing protein 1; FGF1, fibroblast growth factor 1; BAX, Bcl-2-associated X protein; FN1, fibronectin 1; MTBP, MDM2 binding protein; ECT2, epithelial cell transforming 2; GAPDH, glyceraldehyde-3-phosphate dehydrogenase.

LightCycler $^{\circledR} 480$ SYBR Green I Master (Roche Diagnostics), $0.2 \mu \mathrm{l}$ of forward primer, $0.2 \mu \mathrm{l}$ of reverse primer and $3.6 \mu \mathrm{l}$ of nuclease-free water. Reactions were incubated in a 384-well optical plate (Roche Diagnostics) at $95^{\circ} \mathrm{C}$ for $10 \mathrm{~min}$, followed by 40 cycles at $95^{\circ} \mathrm{C}$ for $10 \mathrm{sec}$ and $60^{\circ} \mathrm{C}$ for $30 \mathrm{sec}$. At the end of the PCR cycles, melting curve analysis was performed to validate the specific generation of the expected PCR product. Three independent experiments were performed, with each sample run in triplicate. The primers were synthesized by Generay Biotech Co., Ltd. (Shanghai, China) and are listed in Table II. The expression levels of the genes were normalized with regard to GAPDH and were calculated using the $2^{-\Delta \Delta \mathrm{Cq}}$ method (13).

Cell proliferation, colony formation, invasion and motility assays. Cell viability was determined at 24, 48, 72 and $96 \mathrm{~h}$ using the Cell Counting Kit-8 (Dojindo Laboratories, Kumamoto, Japan), according to the manufacturer's protocol. For the colony formation assay, cells were counted, seeded at low density $(1,000,1,500$ and 2,000 cells/plate), and allowed to grow until visible colonies appeared. Cells were then stained with crystal violet (GenMed ,Shanghai, China) and colonies were counted by eye. In vitro invasion assay was performed using 24-well Transwell units (Corning Life Science, Tewksbury, MA, USA) with polycarbonate filters coated on the upper side with Matrigel (BD Biosciences, Franklin Lakes, NJ, USA). The cells were harvested, and $4 \times 10^{4}$ cells were placed in the upper part of the Transwell unit and were allowed to invade the membrane for $72 \mathrm{~h}$ at $37^{\circ} \mathrm{C}$. Successfully penetrating cells were fixed, stained and quantified at an optical density of $570 \mathrm{~nm}$. The motility assay was conducted in a similar fashion without coating with Matrigel. In total, $8 \times 10^{4}$ cells were seeded per well and incubated in $5 \% \mathrm{CO}_{2}$ at $37^{\circ} \mathrm{C}$ for $5 \mathrm{~h}$ prior to detection. Successfully penetrating cells were quantified at an optical density of $570 \mathrm{~nm}$ using a microplate reader (Infinite 200 Pro; Tecan Schweiz AG, Männedorf, Switzerland). Each experiment was performed in triplicate.

Apoptosis assay. Cell apoptosis was determined by flow cytometric analysis. Briefly, floating and attached cells were collected, resuspended, stained with 
A

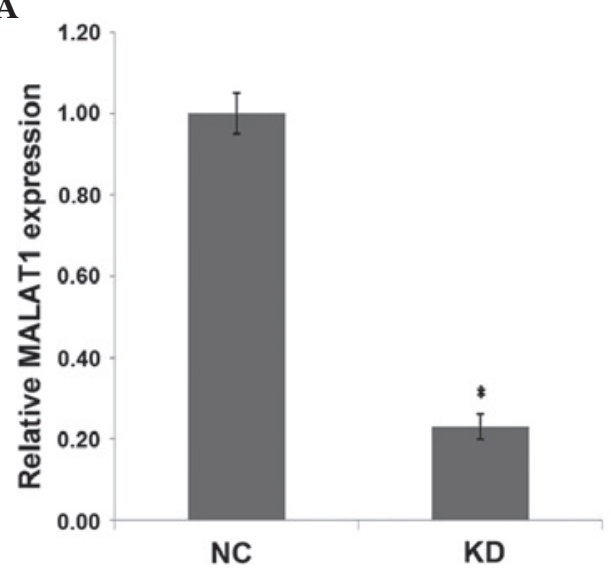

D
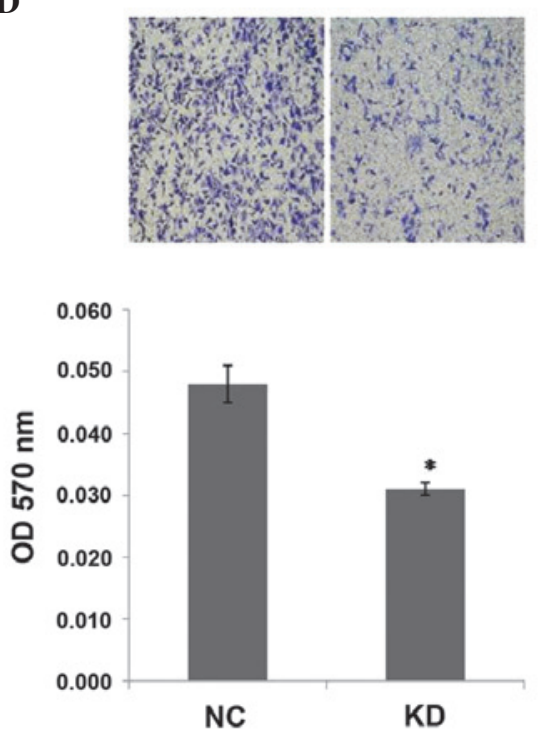

B

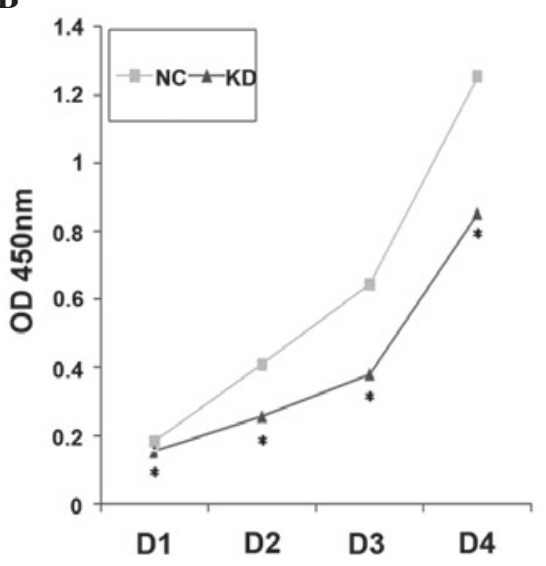

E
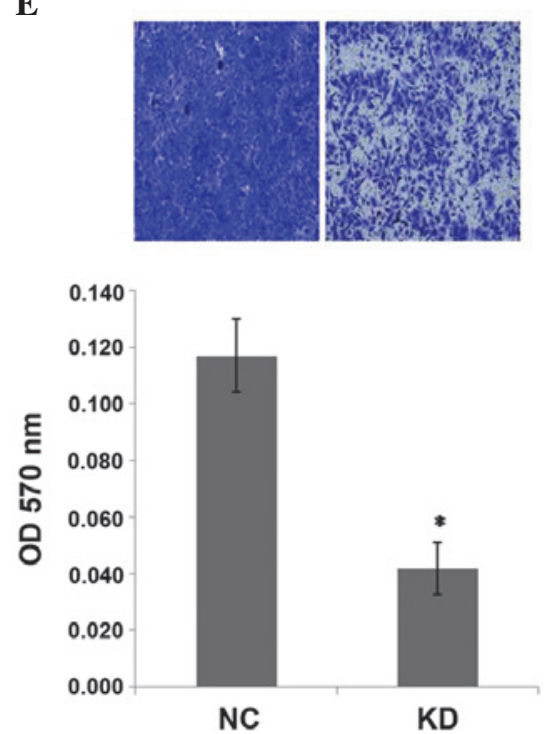

C
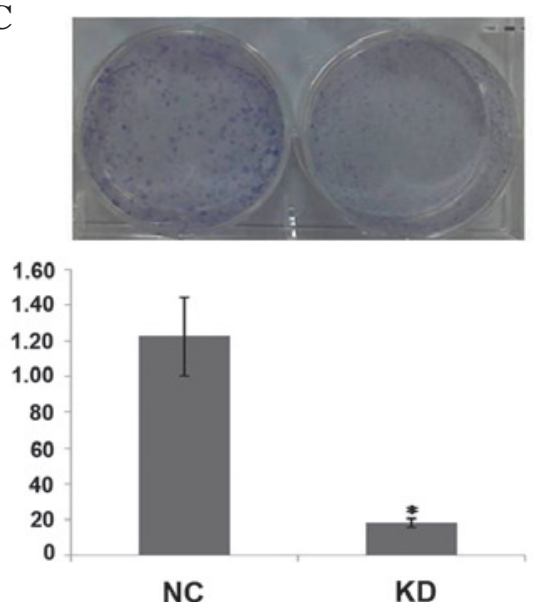

F
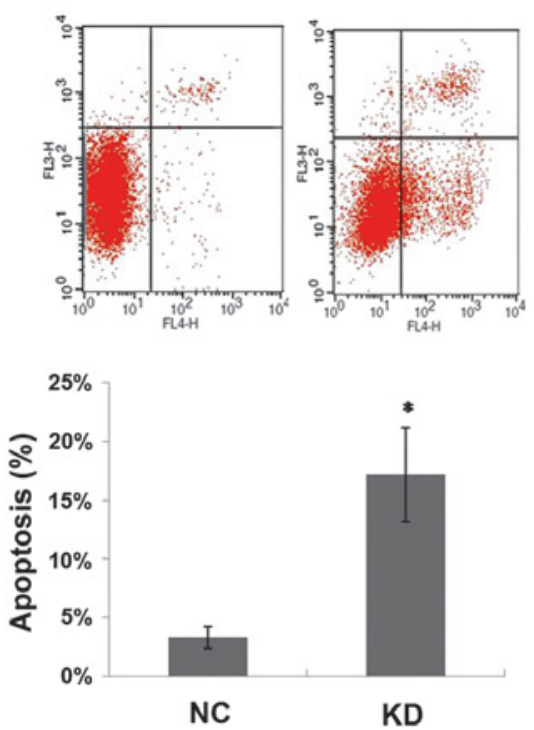

Figure 1. Inhibition of MALAT1 expression suppresses tumorigenicity and induces apoptosis in vitro. (A) Relative MALAT1 expression ( $\mathrm{P}=0.000)$, (B) cell proliferation assay ( $\left.{ }^{*} \mathrm{P}<0.01\right),(\mathrm{C})$ colony formation assay $\left({ }^{*} \mathrm{P}=0.001\right)$, (D) Transwell-migration assay $\left({ }^{*} \mathrm{P}=0.002\right),(\mathrm{E})$ invasion assay $\left({ }^{*} \mathrm{P}=0.001\right)$ and $(\mathrm{F})$ apoptosis assay ( $\left.{ }^{*} \mathrm{P}=0.004\right)$ in $\mathrm{KD}$ cells and NC cells. MALAT1, metastasis-associated lung adenocarcinoma transcript 1; NC, normal control cells; KD, stable MALAT1-knockdown cells; OD, optical density; D1, day 1; D2, day 2; D3, day 3; D4, day 4.

Annexin V-allophycocyanin (BD Biosciences) and incubated for $30 \mathrm{~min}$ in the dark at $20^{\circ} \mathrm{C}$. The analysis was then performed using a BD FACSAria II flow cytometer (BD Biosciences).

In vivo tumorigenic assay. Mice were housed and maintained in a specific pathogen-free facility under controlled environmental conditions (temperature, $22 \pm 2^{\circ} \mathrm{C}$; humidity, $55 \pm 10 \%$ ) with ad libitum access to rodent chow and water. KD cells or NC cells $\left(5 \times 10^{6}\right)$ were injected subcutaneously into the left armpit of nude mice ( 6 to 7 weeks old; $n=7 /$ group). Once palpable tumors developed, caliper measurements were taken twice a week and tumor volume was calculated on the basis of width (x) and length $(y): x^{2} y / 2$, where $x<y$. All mice were sacrificed by cervical dislocation when tumors reached $2 \mathrm{~cm}^{3}$ in size, and tumors were collected.

Microarray analysis and verification of selected genes by $R T$-qPCR. Microarray analysis was performed by Shanghai OeBiotech. Co., Ltd. (Shanghai, China), using SurePrint G3
Human Gene Expression 8x60 K version 2 software (Agilent Technologies, Inc.). Briefly, total RNAs from KD cells and control cells were used to synthesize complementary DNA, from which labeled complementary RNA was then synthesized and hybridized to SurePrint G3 Human Gene Expression 8x60K v2 (Agilent Technologies, Inc.). Subsequent to hybridization, processed slides were washed using the Gene Expression Wash Pack (Agilent Technologies, Inc.) and scanned using the Agilent Microarray Scanner (Agilent Technologies, Inc.). The acquired array images were analyzed using Agilent Feature Extraction software (version 10.7; Agilent Technologies, Inc.), which performs background subtractions. Quantile normalization and subsequent data processing were performed using the GeneSpringGX version 11.0 software package (Agilent Technologies, Inc.). A threshold of a $>2$-fold change was used to screen upregulated or downregulated genes. A total of 20 differentially-expressed genes, which have been reported to be associated with cell proliferation, metastasis and apoptosis, were subsequently selected for validation using RT-qPCR. 
Table III. Deregulated genes in metastasis-associated lung adenocarcinoma transcript 1-knockdown cells compared with negative control cells.

\begin{tabular}{lcrr}
\hline Genbank access number & Gene symbol & Microarray fold change & qPCR fold change \\
\hline NM_003881 & WISP2 & -17.73 & -10.38 \\
NM_024690 & MUC16 & 4.81 & 3.34 \\
NM_014795 & ZEB2 & -5.87 & -2.66 \\
NM_004530 & MMP2 & -5.17 & -3.43 \\
NM_005940 & MMP11 & -4.32 & -5.07 \\
NM_001025370 & VEGFA & -7.93 & -4.92 \\
NM_002632 & PGF & -4.87 & -8.38 \\
NR_002196 & H19 & -4.47 & -3.35 \\
NM_005235 & ERBB4 & -20.57 & -12.87 \\
NM_003068 & SNAI2 & 9.01 & 3.40 \\
NM_024680 & E2F8 & 4.63 & 7.98 \\
NM_001798 & CDK2 & 5.67 & 8.54 \\
NM_001255 & CDC20 & 4.52 & 5.67 \\
NM_000679 & ADRA1B & 4.26 & 7.82 \\
NM_000800 & FGF1 & 4.68 & 5.14 \\
NM_138764 & BAX & 4.48 & 2.05 \\
NM_054034 & FN1 & -23.30 & -3.79 \\
NM_022045 & MTBP & 4.70 & 5.55 \\
NM_018098 & ECT2 & 4.29 & 3.87 \\
\hline
\end{tabular}

qPCR, quantitative polmerase chain reaction; WISP2, WNT1 inducible signaling pathway protein 2; MUC16, mucin 16, cell surface associated; ZEB2, zinc finger E-box binding homeobox 2; MMP2, matrix metallopeptidase 2; MMP11, matrix metallopeptidase 11; VEGFA, vascular endothelial growth factor A; PGF, placental growth factor; H19, H19, imprinted maternally expressed transcript (non-protein coding); ERBB4, erb-b2 receptor tyrosine kinase 4; SNAI2, snail family zinc finger 2; E2F8, E2F transcription factor 8; CDK2, cyclin-dependent kinase 2; CDC20, cell division cycle 20; ADRA1B, adrenoceptor $\alpha$ 1B; FGF1, fibroblast growth factor 1; BAX, Bcl-2-associated X protein; FN1, fibronectin 1; MTBP, MDM2 binding protein; ECT2, epithelial cell transforming 2.

Statistical analyses. All statistical analyses were performed using the SPSS 17.0 software (SPSS Inc., Chicago, IL, USA). Differences between means were analyzed using a two-tailed Student's t-test. $\mathrm{P}<0.05$ was considered to indicate a statistically significant difference.

\section{Results}

KD and NC cells were successfully established. The four pcDNA6.2-GW/EmGFP-miR vectors harboring various miRNAs (KD1, KD2, KD3 and KD4) were used to transiently infect $293 \mathrm{~T}$ cells. In parallel, an NC experiment was performed. The highest knockdown efficiency was achieved using KD3 (71\% relative to the NC group), which was then used for producing recombinant lentiviruses. The results of the RT-qPCR showed that the expression of MALAT1 was decreased by $77 \%$ in $\mathrm{KD}$ cells, compared with $\mathrm{NC}$ cells $(\mathrm{P}=0.000)$ (Fig. 1A).

MALAT1 inhibition suppresses tumorigenicity in vitro. As shown in Fig. 1B, a significant decrease in cell proliferation was observed over time in KD cells compared with NC cells $(\mathrm{P}<0.01)$. MALAT1 inhibition also decreased the clonogenicity of KD cells compared with NC cells $(\mathrm{P}=0.001)$ (Fig. 1C). Transwell migration and invasion assays showed that MALAT1 inhibition decreased the migration $(\mathrm{P}=0.002)$ and invasion $(\mathrm{P}=0.001)$ of KD cells (Fig. 1D and $\mathrm{E})$. These observations suggest that MALAT1 knockdown suppresses the tumorigenicity of ovarian cancer cells in vitro.

MALAT1 inhibition induces apoptosis in KD cells. Apoptosis in KD and NC cells was measured by flow cytometry analysis. The mean apoptotic cell fractions (early apoptotic+apoptotic) were significantly increased upon MALAT1 inhibition compared with the negative control $(\mathrm{P}=0.004)$, with a concomitant decrease in the viable cell population (Fig. 1F). This finding suggests an anti-apoptotic role of MALAT1 in SKOV3 cells.

MALAT1 inhibition suppresses tumor growth in vivo. Since the in vitro data revealed the antitumorigenic role of MALAT1 inhibition in ovarian cancer cells, the effect of MALAT1 inhibition on tumor growth was examined in vivo. As shown in Fig. 2, the mean volume of the tumors in the KD group was significantly smaller compared with the tumors in the NC group $(\mathrm{P}<0.01)$, suggesting that the inhibition of MALAT1 significantly suppressed the tumorigenicity of SKOV3 cells in vivo.

Genes identified during the knockdown of MALATl expression. In order to additionally characterize the function of 

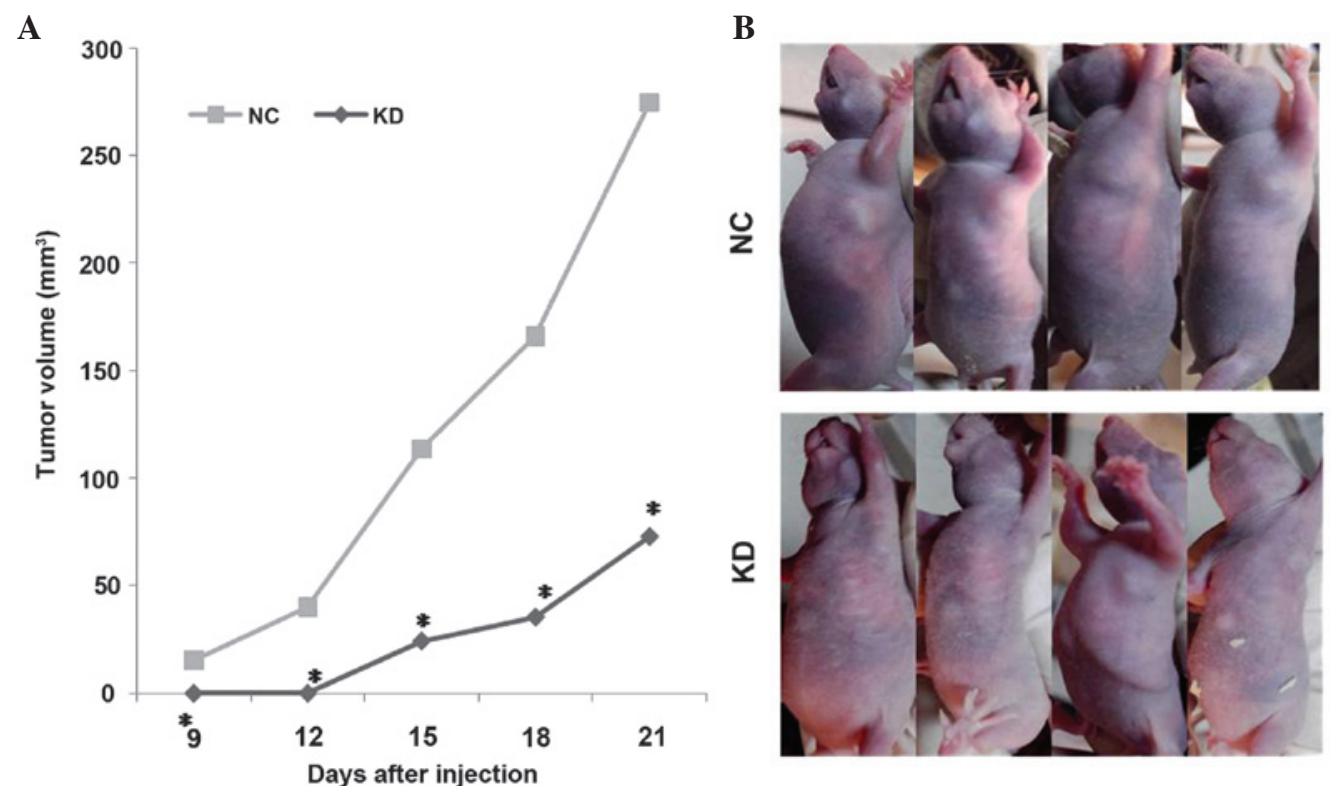

Figure 2. Inhibition of MALAT1 expression suppresses tumor growth in vivo. (A) Mean volume of tumors in the KD group was significantly smaller compared with the NC group. "P<0.01, KD vs. NC. (B) Representative images of mice from the two groups. MALAT1, metastasis-associated lung adenocarcinoma transcript 1; NC, normal control cells; KD, stable MALAT1-knockdown cells.

MALAT1 within cells, RNA isolated from the KD cells was hybridized to the Agilent gene expression microarray and compared with the RNA isolated from the NC group. Data analysis showed that, when compared with the NC cells, a $>2$-fold deregulation was indicated in 921 genes in the KD cells. Since MALAT1 inhibition suppresses tumorigenicity and induces apoptosis in ovarian cancer cells, 20 genes, which have previously been reported to be associated with cell proliferation, metastasis and apoptosis, were selected for validation. As shown in Table III, 19 of these genes confirmed the deregulation that was indicated by the microarray analysis.

\section{Discussion}

MALAT1 was one of the first cancer-associated lncRNAs to be identified, and is widely expressed in normal human tissues. In 2003, Ji et al reported for the first time that MALAT1 was upregulated in non-small cell lung cancer and was a prognostic parameter for patient survival (8). Subsequent studies showed that MALAT1 was also associated with the formation and progression of several other types of tumors (9-12,14-18). However, the role of MALAT1 in ovarian cancer remains unknown.

The present study first investigated the effect of MALAT1 inhibition on SKOV3 cells, and the in vitro and in vivo data suggested that MALAT1 inhibition suppressed tumorigenicity in SKOV3 cells. A limitation to the present study was the lack of clinical samples available. Additional studies with clinical samples are warranted.

The gene expression in KD cells was then compared with control cells, and a large number of genes that were altered in association with decreased MALAT1 expression were identified. The results of the RT-qPCR validation showed that MALAT1 inhibition altered the expression levels of genes that have been reported to be associated with cell proliferation (placental growth factor, cyclin-dependent kinase 2, cell division cycle 20, adrenoceptor $\alpha 1 \mathrm{~B}$ and fibroblast growth factor 1), metastasis [WNT1 inducible signaling pathway protein 2 , mucin 16 , cell surface associated, zinc finger E-box binding homeobox 2 (ZEB2), matrix metallopeptidase 2, matrix metallopeptidase 11, vascular endothelial growth factor A, H19, imprinted maternally expressed transcript (non-protein coding), snail family zinc finger 2 (SNAI2) and fibronectin 1], and apoptosis [erb-b2 receptor tyrosine kinase 4 and $\mathrm{Bcl}-2$-associated $\mathrm{X}$ protein $(B A X)]$. Among these genes, ZEB2, SNAI2 and BAX have been reported to be possible targets of MALAT1 $(10,11)$. The mechanism of regulation of gene expression by MALAT1 remains unclear. Tripathi et al reported that MALAT1 regulated alternative splicing by modulating SR splicing factor phosphorylation (19). However, this phenomenon was not observed in lung cancer cells (20). Additional studies are required in order to clarify the mechanisms underlying MALAT1.

In conclusion, MALAT1 inhibition significantly suppressed tumorigenity in ovarian cancer cells and the inhibition of MALAT1 altered the expression of a number of genes associated with cell proliferation, metastasis and apoptosis. Overall, the findings of the present study define a major oncogenic role for MALAT1, which may provide an attractive novel target for therapeutic intervention in ovarian cancer.

\section{Acknowledgements}

The present study was supported by the National High Technology Research and Development Program of China, Ministry of Science and Technology of the People's Republic of China, Beijing, China (grant no., 2012AA02A507). 


\section{References}

1. Siegel RL, Miller KD and Jemal A: Cancer statistics, 2015. CA Cancer J Clin 65: 5-29, 2015

2. Holschneider $\mathrm{CH}$ and Berek JS: Ovarian cancer: Epidemiology, biology, and prognostic factors. Semin Surg Oncol 19: 3-10, 2000.

3. Mørch LS, Løkkegaard E, Andreasen AH, Krüger-Kjaer S and Lidegaard O: Hormone therapy and ovarian cancer. JAMA 302 298-305, 2009.

4. Lin HW, Tu YY, Lin SY, Su WJ, Lin WL, Lin WZ, Wu SC and Lai YL: Risk of ovarian cancer in women with pelvic inflammatory disease: a population-based study. Lancet Oncol 12:900-904, 2011.

5. Rinn JL, Kertesz M, Wang JK, Squazzo SL, Xu X, Brugmann SA, Goodnough LH, Helms JA, Farnham PJ, Segal E and Chang HY: Functional demarcation of active and silent chromatin domains in human HOX loci by noncoding RNAs. Cell 129: 1311-1323, 2007.

6. Wang X, Arai S, Song X, Reichart D, Du K, Pascual G, Tempst P, Rosenfeld MG, Glass CK and Kurokawa R: Induced ncRNAs allosterically modify RNA-binding proteins in cis to inhibit transcription. Nature 454: 126-130, 2008.

7. Ogawa Y, Sun BK and Lee JT. Intersection of the RNA interference and X-inactivation pathways. Science 320: 1336-1341, 2008.

8. Ji P, Diederichs S, Wang W, Böing S, Metzger R, Schneider PM, Tidow N, Brandt B, Buerger H, Bulk E, et al: MALAT-1, a novel noncoding RNA, and thymosin beta4 predict metastasis and survival in early-stage non-small cell lung cancer. Oncogene 22: 8031-8041, 2003.

9. Lai MC, Yang Z, Zhou L, Zhu QQ, Xie HY, Zhang F, Wu LM, Chen LM and Zheng SS: Long non-coding RNA MALAT-1 overexpression predicts tumor recurrence of hepatocellular carcinoma after liver transplantation. Med Oncol 29: 1810-1816, 2012.

10. Guo F, Li Y, Liu Y, Wang J, Li Y and Li G: Inhibition of metastasis-associated lung adenocarcinoma transcript 1 in CaSki human cervical cancer cells suppresses cell proliferation and invasion. Acta Biochim Biophys Sin (Shanghai) 42: 224-229, 2010.
11. Ying L, Chen Q, Wang Y, Zhou Z, Huang Y and Qiu F: Upregulated MALAT-1 contributes to bladder cancer cell migration by inducing epithelial-to-mesenchymal transition. Mol Biosyst 8: 2289-2294, 2012.

12. Yang MH, Hu ZY, Xu C, Xie LY, Wang XY, Chen SY and Li ZG: MALAT1 promotes colorectal cancer cell proliferation/migration/invasion via PRKA kinase anchor protein 9. Biochim Biophys Acta 1852: 166-174, 2015.

13. Livak KJ and, Schmittgen TD: Analysis of relative gene expression data using real-time quantitative PCR and the 2(-Delta Delta C(T)) Method. Methods 25: 402-408, 2001.

14. Pang EJ, Yang R, Fu XB and Liu YF: Overexpression of long non-coding RNA MALAT1 is correlated with clinical progression and unfavorable prognosis in pancreatic cancer. Tumour Biol 36: 2403-2407, 2015.

15. Zhang HM, Yang FQ, Chen SJ, Che J and Zheng JH: Upregulation of long non-coding RNA MALAT1 correlates with tumor progression and poor prognosis in clear cell renal cell carcinoma. Tumour Biol 36: 2947-2955, 2015.

16. Dong Y, Liang G, Yuan B, Yang C, Gao R and Zhou X: MALAT1 promotes the proliferation and metastasis of osteosarcoma cells by activating the PI3K/Akt pathway. Tumour Biol 36: 1477-1486, 2015.

17. Cho SF, Chang YC, Chang CS, Lin SF, Liu YC, Hsiao HH, Chang JG and Liu TC: MALAT1 long non-coding RNA is overexpressed in multiple myeloma and may serve as a marker to predict disease progression. BMC Cancer 14: 809, 2014.

18. Wang J, Su L, Chen X, Li P, Cai Q, Yu B, Liu B, Wu W and Zhu Z: MALAT1 promotes cell proliferation in gastric cancer by recruiting SF2/ASF. Biomed Pharmacother 68: 557-564, 2014.

19. Tripathi V, Ellis JD, Shen Z, Song DY, Pan Q, Watt AT, Freier SM, Bennett CF, Sharma A, Bubulya PA, et al: The nuclear-retained noncoding RNA MALAT1 regulates alternative splicing by modulating SR splicing factor phosphorylation. Mol Cell 39: 925-938, 2010.

20. Gutschner T, Hämmerle M, Eissmann M, Hsu J, Kim Y, Hung G, Revenko A, Arun G, Stentrup M, Gross M, et al: The noncoding RNA MALAT1 is a critical regulator of the metastasis phenotype of lung cancer cells. Cancer Res 73: 1180-1189, 2013. 